\title{
Probabilistic Models for Drug Dissolution. Part 1. Review of Monte Carlo and Stochastic Cellular Automata Approaches
}

\author{
Ana Barat \\ Heather J. Ruskin \\ Martin Crane \\ School of Computing, Dublin City University, Dublin 11, Ireland
}

\begin{abstract}
Throughout the last decades, Monte Carlo (MC) techniques have been used in simulating various complex systems. In this paper, we investigate how MC-based methods are used in the field of Drug Delivery, indicating what aspects of the complex problems of drug dissolution and design can benefit from this particular approach. After introducing the area of modelling drug dissolution, with its different features and needs, we report and examine the existing Direct MC and Stochastic Cellular Automata modelling efforts used to simulate dissolution of pharmaceutical compacts or related phenomena. In Part 2, we enlarge on a description of our work on Direct MC, for the particular case of simulating a binary system consisting of poorly soluble drug dispersed in a matrix of highly-soluble acid excipient.
\end{abstract}

KEYWORDS

Modelling; Drug Delivery Systems; Drug Dissolution; Drug Release; Design and Experiment; Multicomponent systems; Monte Carlo; Cellular Automata; Porosity; Dissolution through pores.

\section{Introduction to Modelling Drug Dissolution}

Pharmaceutical Drug Delivery Systems (DDS) are systems which deliver a drug into the body. A DDS consists of one or several bio-active agents (the drug(s)), and one or several excipients, which are substances forming the vehicle or medium for the administration of the active agents. The dissolution profile of the drug molecule can be controlled with a wide range of excipients.

The ultimate aim of a DDS is to undergo dissolution in a biological medium (eg.: gastrointestinal tract for tablets, biological tissues for implants etc.) and, as released, to undergo absorption. The release of the drug and thus, the ultimate absorption, must take place at appropriate rates, in order to ensure the desired effect on the organism. In many drug delivery applications, the release of the excipient (involving diffusion, dissolution, erosion or other mechanisms) determines the release of the bio-active agents. Some modern formulations are very complex, and the relationship between the formulation and process variables and the release profiles are not entirely understood.

From performance considerations, it is important to investigate the effects of factors related to the composition, design and manufacturing process [14], on the phenomenological behaviour of a drug delivery system. In vitro dissolution testing is very important in terms of designing, developing and testing new formulations [35, 10], and it can be regarded as the first step to modelling the dissolution in vivo. The dissolution profile in vitro has to satisfy certain criteria established by the pharmacopoeias, in order to maintain the in vivo drug release at the desired rate.

Depending on the design and delivery goals of a particular DDS, the process of formulation development and optimisation may require a significant amount of time and effort, as well as financial investment [36]. The direct benefit of producing accurate models for in vitro dissolution is reduction in the laborious drug formulation studies and the minimisation of costs associated with them. This can be achieved by investigating how design parameters such as composition, 


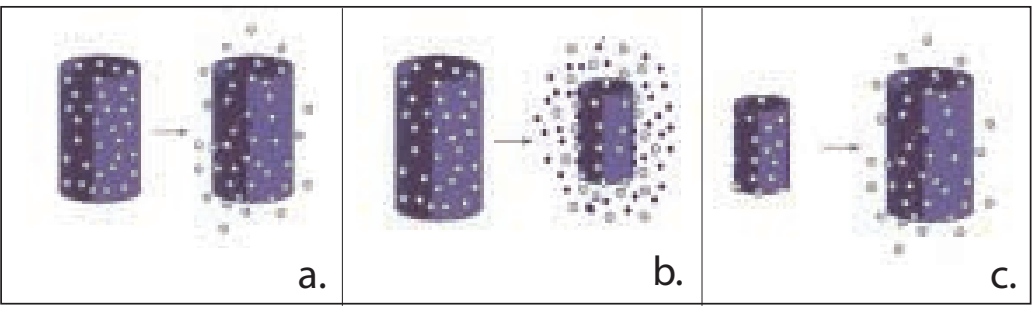

Figure 1: Schematic representation of behaviours of different types of DDS, where the excipients are: a) inert matrix. b) bio-erodible. c) swellable.

porosity and device geometry, affect the resulting kinetics of drug release [35] and by selecting only the most interesting candidate parameters to perform in vitro experimentation [44]. In addition, modelling can lead to developing better scientific understanding of the behaviour of a number of studied systems.

The following section briefly discusses problematic aspects associated with the field of drug dissolution, and indicates a number of different modelling approaches taken to provide insights in this active research area.

In the drug development field various materials, manufacturing techniques and release mechanisms are used to achieve different drug release profiles. The mass transport mechanisms involved include, but are not limited to: diffusion, dissolution, the combination of diffusion and dissolution, water triggered transport (swelling), degradation/erosion, as well as phenomena intrinsic to osmotic, magnetic or electric effects [32].

For example, a water soluble drug, incorporated in a hydrophobic, inert matrix system with channels and pores (e.g. ethylcellulose, hydrogenated castor oil), is mainly released by diffusion through the polymer into the surrounding medium, Figure 1, (a). The release rate of the incorporated drug will depend not only on the particle size and the particle size distribution of the ethylcellulose used, but also on processing variables, like the compaction pressure used while manufacturing (which results in larger or smaller pores in the matrix).

In other drug delivery systems where the main release mechanism is diffusion, the release rate of the drug is maintained as desired by the suppressing or enhancing effect of an excipient.

In the drug delivery, primarily controlled by the erosion of the excipient, consisting of bioerodible polymers, the self-erosion of the matrix is the principal release mechanism [8]. The erosion behaviour of the used materials, available in various forms (e.g. different molecular weights), is a crucial factor in the device performance. All bio-erodible polymers have characteristic times of erosion. This permits formulation of laws describing the erosion and prediction of monomer or drug release, Figure 1, (b). Description of this kind of DDS are given in [33].

Some of the the most complex examples of drug delivery systems are those controlled by hydrophilic swellable/erodible excipient, behaving as a reservoir-type controlled-release device, ${ }^{1}$ Figure 1, (c). The most important characteristic of this kind of system is that, by diffusion of water into the hydrophilic polymeric matrix, the compact swells, radically changing its form and dimensions. The water-soluble incorporated molecules of drug diffuse both within and out of the device, through the disentangled chains of polymer [35, 34].

These and other phenomena, like superposition, synergies or coexistence of these physicochemical effects, should be taken into consideration when producing models.

The aforementioned also illustrates that the topic of drug release is subject to considerable diversity, so that modelling and simulating the process of drug release has many ramifications. In seeking a better understanding of the phenomena involved in mass transport, different modelling approaches have been taken into consideration throughout the last decades.

\footnotetext{
${ }^{1} \mathrm{~A}$ DDS with the aim of maintaining an uniform dose over a specified period of time
} 
The literature presents a wide range of models - from simple ones: such as the empirical and semi-empirical, to more complex: such as those involving mechanistic theories. ${ }^{2}$ In contrast to mechanistic mathematical models, empirical models quantifying drug release from delivery systems are not so obviously based on the exact description of all the real physical processes involved. Empirical models can only describe the resulting, apparent drug release rates [33].

In order to build a mechanistic model, one has to be aware of both the major governing processes within the system and the basic mass transport mechanisms involved in drug release. Such models should be able to provide knowledge about the system's behaviour and are generally powerful with respect to simulating the effect of the device design variables on the resulting drug release kinetics [33]. Most existing mechanistic models are based on mathematical equations, describing the mass transport phenomena which take place while drug is released. A spectrum of mathematical theories, describing drug release from controlled/immediate-release delivery systems, is available [32]: for reviews see [35, 33, 29, 8].

However, most of the present day pharmaceutical compacts typically show much more complex behaviour than those initially modelled by the non-linear mathematical equations approach, and have few simplifying properties which permit the use of non-linear dynamics. The whole picture is made even more complex by the presence of one or more moving boundaries, as the components dissolve. In addition, for some systems, it is not a trivial task to identify the most important factors, among both the formulation components and process parameters, that should be considered to better model the drug release.

In an effort to capture different aspects related to the growing complexity in the drug delivery field, recently the literature has shown a trend in the development of less traditional methods which consider alternative ways of looking at the drug dissolution. For example the stochastic approaches [5], Cellular Automata and direct Monte Carlo methods (see below) are based on the idea that the drug release process is often subject to random fluctuations. The advantage of the later is the possibility of making a simplified, but direct representation of the studied system, which gives the possibility of monitoring its evolution with the time, in the effort to understand the importance of a number of microscopic effects. These methods are especially interesting to consider when modelling porous compacts with complex and non-homogeneous inner structure, like mixtures of species exhibiting various particle sizes and very different behaviours when exposed to a dissolution medium. Methods for optimisation in drug design based on artificial neural networks and genetic algorithms are presented in $[39,42,41,6,40]$. When the relationship between the drug release profiles and the formulation and process factors is not linear and, moreover, is not well understood, the artificial neural networks have the advantage of mapping the relationship between the variables and the responses, such as drug release profiles, through learning or training processes [39].

\section{Introduction to direct Monte Carlo methods}

Monte Carlo (MC) methods are algorithms used for solving various kinds of computational problems using random numbers (or rather pseudo-random numbers), in a sampling procedure. Such methods contrast with deterministic algorithms, like molecular dynamics or direct numerical solutions (eg.: Finite Elements, Finite Differences etc.) by being stochastic, that is non deterministic, [1]. MC methods are rooted in concepts of statistical randomisation and sampling ${ }^{3}$ and were first used to address many-body problems in the middle of the 20th Century.

$\mathrm{MC}$ is extremely important in computational physics and related applied fields, because phenomena, which are otherwise difficult to quantify, can be treated as distributions of random num-

\footnotetext{
${ }^{2} \mathrm{~A}$ mechanistic model should have as many features, of the primary system built into it, as observations and data allow; that is, the model should be consistent with the observed behaviour of the system and be predictive about its future behaviour under the effect of perturbation, [3].

${ }^{3}$ Sampling experiments are based on generation of random numbers, followed by various arithmetic and logical steps, which are often highly repetitive, hence highly amenable to handling by computer. In the same ways, the power of the methods, which contain many variables, has grown with the increase in computer power.
} 
bers. The following studies $[19,20,28,23]$ and references therein discuss both direct and inverse Monte-Carlo simulations, as well as a broad range of applications.

Direct MC simulations, applied to multiple-particle systems, have been frequently used to mimic various problems from real life systems. Earlier examples of problems, which direct Monte Carlo techniques have been applied to, include the study of systems of interacting atoms, the study of radioactive decay, the simulation of a fluid flow from an atomistic perspective, the simulation of traffic on roads etc.

While modelling systems exhibiting complex behaviour, often direct MC sampling methods are used in the framework of Cellular Automata. Cellular Automata are defined in [7] as an idealisation of a physical system in which space and time are discrete, and the physical quantities take only a finite set of values. This basically consists of representing the system as a computational grid with specified local relationships, in order to record any prior knowledge or theories about the microscopic transient behaviour of the real system (such as, for eg., the intrinsic dissolution rates of the components belonging to a DDS, dissolution rules etc [44]). When considering the transient behaviour of a system, modelled in the framework of Cellular Automata, one or several dynamic particularities can be treated as deterministic or, conversely, stochastic, with sampling based on Monte Carlo techniques. The second idea is obviously applied when the modeller does not have exact or complete knowledge about the real system, which is often the case.

By allowing the model to evolve according to the stochastic relations (or rules) specified, then statistically averaging over a number of such samples, it is possible to mimic the global, macroscopic behaviour of the system. Thus, predictions can be made about the system's behaviour as a function of the initial parameters. This permits to explore the way, in which microscopic behaviour of system components gives rise to important macroscopic features and emergent behaviours.

The primary objective of this review and accompanying paper, is investigating the application of MC methods to drug dissolution. For this reason, in the following section we present a review of the most relevant examples from literature, where direct MC simulations were used to study the behaviour of drug delivery, or other multi-particle systems, which bear some similarity to the systems which interest us.

\section{Review of dissolution problems, addressed by Direct Monte Carlo techniques}

\subsection{Monte Carlo methods for dissolution of various types solids}

Crystalline solids are arranged in fix geometric patterns or lattices. They have orderly arranged units and are practically incompressible. On the other hand, amorphous solids are solids with randomly oriented molecules. They are supercooled liquids where the molecules are arranged in a random manner. Both crystalline and amorphous solids are used in drug manufacture. We discuss the literature of examples of use in the following sections.

\subsubsection{Polycrystalline solid}

A direct MC method has been used by Srinivasan [37], to simulate the micro-structural evolution during the dissolution of a polycrystalline solid. The 2D or 3D hexagonal grid for computation represents a labelled region of circular or spherical solid, placed in the middle of a labelled area of liquid with sink ${ }^{4}$ properties. The dissolution is accounted for by:

- considering that the "solid" grid sites are unstable.

- associating with each of them a value of free energy.

- assuming that the liquid phase is stable and has a free energy equal to zero.

\footnotetext{
${ }^{4}$ The liquid is considered to be infinite. No matter how much solute it accepts, the concentration of the solute in the sink liquid is set to zero.
} 
- allowing the dissolution process to occur in the direction of the decreasing free energy and

- allowing reverse reaction.

The probabilistic MC process is carried out by selecting a cell at random and allowing it to change to liquid state. If there is a decrease in the total energy associated with the cell, it is allowed to stay in the liquid state, if not, it reverts to the solid state. This model permitted a parametric study of the influence of interface energies on the dissolution of the solid.

\subsubsection{Glass}

Another model, which investigates glass dissolution in water, by Santra et al. [31], treats the solid (i.e. glass) like a random binary system with strongly different solubilities. One of the two species has a local environment-dependent solubility. The model also permits for re-crystallisation of the components. For a finite volume of glass exposed to the liquid, a rapid dissolution is observed, until an apparent pseudo-equilibrium is reached, with a steady-state of glass porosification continuing at a lower, but constant speed. Porosification is a process which occurs during drug dissolution from some pharmaceutical compacts as well. In the case of a porous solid dissolving in a liquid, the solid-liquid interface can be viewed as an irregular area of fractal nature, rather than a 1D linear front. It can occur that dissolution through pores is different than dissolution at the surface. By directly representing such physical situations, Cellular Automata direct MC techniques can directly model these situations, affirming in this way their advantage over differential equationbased methods in modelling porosification. The model above has, for example, permitted a better understanding the physical process of glass dissolution.

\subsubsection{Calcite}

A kinetic Monte Carlo technique which simulates the dissolution and growth of calcite in flowing water is described by Williford et al. [43]. Boundary layer problems are taken into consideration and the diffusion in the fluid is treated by a random walk sub-model. The model is divided into a grid, where each square cell is one calcite lattice unit and represents a site. Each site can be occupied by either a solid phase or by molecules that have been desorbed.

\subsection{Monte Carlo Methods for Controlled Release Applications of Poly- mers}

Controlled release applications are drug delivery applications where the drug has to be released according to a desired profile, usually extremely slowly. This is achieved, for example, by incorporating the drug into slowly eroding polymers. As mentioned previously in Section 1, the systems formed have quite complex behaviours, not trivial for mechanistic modelling.

\subsubsection{Bio-erodible polymer matrix}

Göpferich and Langer [14] have attacked the problem of monomer release from bio-erodible polymer matrices using a Monte Carlo - mechanistic method.

- The structural problem is addressed by Monte Carlo.

- The dynamics are accounted for both by differential equations and Monte Carlo.

The MC part is based on the fact that the polymer erosion is treated as a random phenomenon: the hydrolysis of chemical bonds is modelled by a Poisson process. If several Poisson processes are proceeding in parallel, then the whole process is again a Poisson process. This is the case here with the cleavage of the polymeric bonds [13]. The polymer is represented [14] as a lattice of sites. Each

site represents polymer excipient in a continuous state. A random life-time is assigned to each. The life-times of the sites are random numbers derived using a first order Erlang distribution: 


$$
e(t)=\lambda e^{-\lambda t}
$$

where $\lambda$ is the erosion rate constant and $e(t)$ is the probability that the polymer will erode completely at time $t$, after its first contact with the water. In practice, the time $t$ is computed using the following relation:

$$
t=\frac{1}{\lambda n} \ln (1-\varepsilon)
$$

where $n$ is the grid size and $\varepsilon$ is a random number, uniformly distributed between 0 and 1 , [13]. When one of these sites comes into contact with the buffer solution, its life-time begins to decrease. When it reaches zero, the polymer is considered to have spontaneously degraded to a crystallised monomer. The process of monomer mass loss can begin. This is where the mechanistic part of the model can be introduced. Mass loss takes place by monomer diffusion inside the matrix, described by an equation derived from Fick's first law (see [9]), which accounts for the porosity in the material:

$$
\frac{\partial}{\partial t}[C(x, t) \epsilon(x, t)]=\frac{\partial}{\partial x}\left[D_{e f f}(C) \varepsilon(x, t) \frac{\partial C(x, t)}{\partial x}\right]
$$

Here, $C(x, t)$ is the concentration of diffusing monomer, $\varepsilon(x, t)$ is the porosity and $D_{\text {eff }}(C)$ is the effective diffusion coefficient. As there is no prior knowledge about the porosity, a probabilistic process is used to calculate the porosity along the diffusion pathway. Further, the function $\varepsilon(x, t)$ is built in the following way: the value 1 represents "eroded" and 0 - "non-eroded" sites. Moreover, the authors have included in the model equations accounting for:

- the velocity of dissolution of the monomer (function of the amount of suspended monomer in the solution).

- the $\mathrm{pH}$ effects.

- the boundary conditions.

This model has helped to compute the dissolution rates of monomer. They agreed very well with the experimental dissolution rates. In addition, the model confirmed a previous hypothesis, that the quite unexpected dissolution profiles obtained in the experimental part were due to $\mathrm{pH}$ effects inside the pores. Other authors, like those in $[30,17]$ discuss as well the influence on the overall release kinetics of the $\mathrm{pH}$ inside the pores .

\subsubsection{Layered bio-erodible polymer matrix filled with drug}

In [12], Göpferich uses a similar model for a slightly different situation. The problem is to model matrices consisting of several layers of two different erodible polymers (polyanhidride and poly(D,L-lactic acid)). Only one of these polymers is loaded with drug. It has to release the drug in two different phases, due to the layers. To address this, the author considered two different species of polymer sites (pixels): crystalline (pure polymer) and amorphous (loaded with drug), with individual erosion rate constants $\lambda_{c}$ and $\lambda_{a}$ respectively. The lifetime $t$ is, on average, higher for crystalline pixels because of their smaller erosion rate $\lambda_{c}$. The release of drug was predicted by assigning to each amorphous pixel a relative amount of drug $\frac{1}{n_{x} n_{y}}$, where $n_{x}$ and $n_{y}$ are the dimensions of the computational grid. It was assumed that, whenever a polymer site erodes, an appropriate amount of hydrophilic drug is spontaneously released directly into the solution. Figure 2 shows the author's way of representing a 3D cylinder in 2D: the grid divides the cross section into individual polymer pixels of the same volume. This is achieved by decreasing the length $\Delta x$ of the sites from the grid, in the radial direction, with the root of the increasing radius. Extensions to $3 \mathrm{D}$ of this model also exist [11]. 


\subsubsection{Bulk-eroding system}

A problem of controlled release devices containing erodible polymers with completely different mechanisms of erosion (surface erosion and bulk erosion) has been solved by a MC approach as well, [13]. The matrix is composed of several layers of polymers, characterised by different erosion mechanisms (note that the previous model worked only in the case of the same mechanism, and two different erosion rates). The layers are as follows:

- The surface-eroding ${ }^{5}$ core is charged with drug.

- The mantle is charged with drug free bulk-eroding polymer. ${ }^{6}$

- The surface-eroding crust is loaded with drug.

In the model, the following distinction is made between surface eroding polymer layers and bulk eroding layers, in order to simulate their different erosion behaviour:

- A surface-eroding polymer site begins to erode only when it comes into contact with the buffer solution.

- Conversely, all the sites of a bulk-eroding polymer have a chance to begin their erosion, as soon as at least one site, (representing the bulk-eroding polymer), comes into contact with the erosion medium.

\subsubsection{Generalisation moldel for bio-erodible systems}

A new type of Cellular Automata method to simulate release from bio-erodible devices, in order to design formulations with optimal release characteristics was discussed by Zygourakis and Markenscoff [44]. The DDS of any shape and the liquid around it is represented as a computational grid, which is designed as a dynamic system with transient behaviour. The microscopic mechanisms of the dissolution phenomena are defined in terms of local relations between the sites. The model can accept two or three solid components, related to amorphous or crystalline bio-erodible polymers, characterised by different erosion rates. What makes this model different from the previous ones from this subsection, is that there are no differential equation used here, and the quantities of matter are treated as discrete. The erosion rates can be made dependent on the micro-environment of the sites concerned.

Possible porosity of the device, with various sizes of the pores is taken into account. The authors discuss methods for time normalisation together with averaging and normalising the dissolution rates, in order to be able to compare the simulation results with experimental data. The simulation results are analysed to show how the overall release rates are affected by the intrinsic dissolution rates, drug loading, porosity and the dispersion of the drug in the bio-erodible matrix. The article suggests that a drug design approach which combines computer simulations and laboratory experimentation is likely to significantly reduce laboratory experimentation and the associated time and costs.

\subsubsection{Swellable systems}

As mentioned is the previous section, the controlled release devices made of swellable polymer seem to have the most complex behaviour of all.

Lee and Chakraborty [26] studied the diffusion of heteropolymers through random disordered media at a very microscopic level, using kinetic Monte Carlo methods. They have investigated the

\footnotetext{
${ }^{5}$ In this case, polymer degradation is much faster than water intrusion in the polymer bulk, so that degradation occurs mainly at the outermost polymer layers. This is why erosion affects only the surface and not the inner parts of the matrix [33].

${ }^{6}$ Bulk eroding polymers erode slowly and water uptake by the system is much faster than polymer degradation. In this case, erosion is not restricted to the polymer surface, because the entire system is rapidly hydrated and polymer chains are cleaved throughout the device [33].
} 


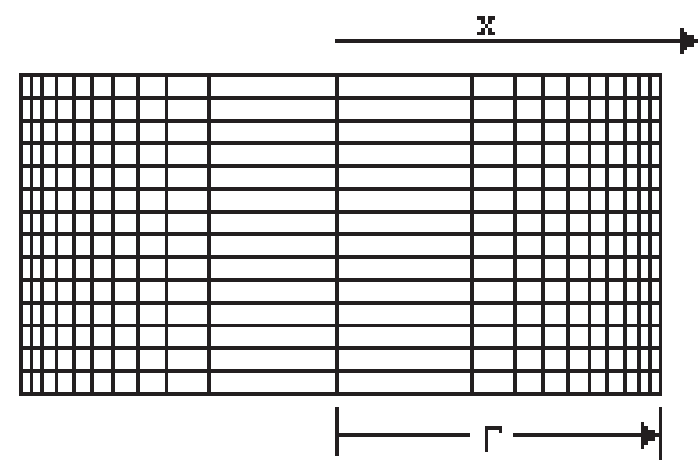

Figure 2: Computational grid to simulate a $3 \mathrm{D}$ cylinder in 2D

possible interactions of the polymeric chain with the fixed obstacles constituting a medium. The study of the polymer behaviour is relevant to understanding the behaviour of swellable compacts, (see Section 1). In controlled release applications of polymers, a solute is molecularly dispersed in a polymer phase. In the presence of a thermodynamically compatible solvent, swelling occurs and the polymer begins to release its contents to the surrounding fluid [29]. The kinetics of the drug release process can be decisively affected by the polymer swelling, due to the external penetrant uptake [15]. This release process can be controlled either by solute diffusion or by polymer dissolution. In this case the presence of permanent entanglements in the polymers becomes significant [29].

In the model of Lee and Chakraborty [26] the polymer is modelled by a chain of spherical beads placed and equilibrated on a 3D lattice partitioned into sites. The lattice sites can be occupied by spherical obstacles. Each MC step consists of an attempt to move chosen beads from the chain. Each movement is accepted or rejected according to the Metropolis criterion, with probability depending on the quantity $\triangle U$. This quantity represents the energy between the old and new chain position. The simulations resulted in a number of interesting effects. For example, the authors found that above a threshold temperature, polymers containing monomers which are attracted to sites from the medium are more mobile than those which exhibit repulsive or neutral interactions. The effect is found to be related to the construction of the disordered medium. This study permitted a better understanding of how polymer sequence influences the dynamics of polymers in quenched disordered media.

\subsection{Monte Carlo in the study of the design parameters for drug delivery systems}

Often, the semi-empirical Weibull function is chosen to fit different kinds of dissolution data, because it provides the best fit to the simulated data. Equation (4) shows the Weibull function, with $a$ and $b$ - constants. These constants are believed to be somehow connected to the shape of the releasing device, [2].

$$
\frac{M_{t}}{M_{\infty}}=1-e^{\left(-a t^{b}\right)}
$$

Recently, Kosmidis et al. [23] have used MC methods to model the drug release from various cylindrical and spherical matrices. The practical advantage of Monte Carlo simulations, as opposite to performing actual experiments, is that they help to produce quickly and for small costs "dissolution" data from many initial shapes of "the device". The authors investigated the physical significance of the parameters of the Weibull function, fitting it to the results of their MC simulations. 
In the model from Kosmidis et al. [23], the drug delivery device is simulated by labelling the desired shape on a computational grid. Each site of the grid, belonging to the device, can accommodate certain numbers of particles. Diffusion is achieved by randomly picking a particle on the grid and moving it to a random nearest-neighbour site (as defined for a von Neumann neighbourhood). Each decision in the simulated system dynamics is taken by drawing random numbers from a uniform distribution. Further, each decision corresponds to an arbitrary time-unit (Monte-Carlo step), which can be shown to correspond to a real-time unit [23]. After specifying, for example, cylindrical or spherical configurations inside their computational grids, "leak sites" are designed at the surface exposed to the solvent. These sites are coupled to the rest of the sites of the device and, hence, particles trapped into the device can diffuse to the release medium. A particle is discarded from the system as soon as it arrives at a "leak site".

As specified, this model would approximate closely the behaviour of a non-eroding polymeric system, where the drug molecules diffuse inside the device according to the concentration gradients, and eventually escape from the device. The dissolution of micro-capsules coated with polymer like those described in [2] are good candidates for being analysed with the model above. The "leak sites" would correspond to the micro-pores formed at the contact with the release medium, in the coating film covering the microcapsules.

The results from the MC simulations [24] has been used to assess the effect of different initial geometrical shapes on the dissolution. The simulated data have been fitted by a Weibull function. Goodness of fit has been evaluated, in order to obtain physical insight for the meaning of the Weibull parameters $a$ and $b$. The authors [24] found linear dependence between these parameters and the expression $\frac{N_{\text {leak }}}{N_{\text {total }}}$. Here $N_{\text {leak }}$ is the number of "leak" sites designated on the surface and $N_{\text {total }}$ is the total number of sites belonging to the device. In fact, $\frac{N_{\text {leak }}}{N_{\text {total }}}=\frac{S}{V}$, where $S$ is the active diffusion surface and $V$ is the volume of the device.

The authors plot both Weibull parameters versus $\frac{N_{\text {leak }}}{N_{\text {total }}}$ on a $2 \mathrm{D}$ axis-plan, labelling the points according to the initial concentration of the drug in the device and the shape of device. Both $a$ and $b$ are found to be independent of the initial concentration of drug in the device. Instead, the geometry of the device seems to have an influence on $b$. In the end, the following equation has been deduced:

$$
\frac{M_{t}}{M \infty}=1-\exp \left(-0.28 \triangle l \frac{S}{V}\left(\frac{t}{\tau}\right)\right)\left(0.65+0.4 \triangle l \frac{S}{V}\right)
$$

Assuming that the drug inside the device undergoes classical Fickian diffusion and expressing

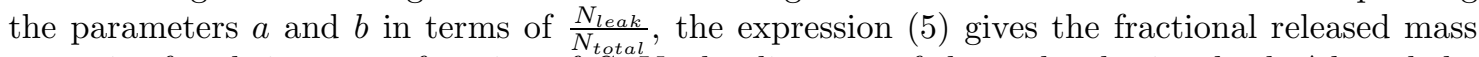
per unit of real time, as a function of $S, V$, the diameter of the molecules involved, $\triangle l$, and the mean free time $\tau^{7}$. The disadvantage of the expression 5 is that $\Delta l$ and $\tau$ usually remain unknown simply because determining their values is a quite difficult task. The conclusions drawn from using MC techniques are similar to those from Antal et al. [2] , who describe a more mathematical approach to explaining the meanings of the Weibull parameters.

\subsection{Monte Carlo in studying fractals and percolation}

A fractal dimension is a very suitable concept to describe a complex structure, (e.g.: an internal surface of a porous system, the roughness of a particle or of a surface, an organ of interest (e.g. liver, lungs, a tumour and so on). Percolation theory is a statistical theory, which deals with the formation of clusters, and supposes the existence of a regular lattice underlying the system [4]. Percolation processes are those in which, by the random addition of a number of objects, a contiguous path which spans the entire system is created [25]. Leuenberger et al. [27] introduced percolation theory in the field of pharmaceutics to explain the mechanical properties of certain

\footnotetext{
${ }^{7}$ The mean free time reflects the particle's average speed, which is directly connected to the temperature of the system.
} 
controlled release compacts. These authors first considered a compact as a disordered system consisting of particles distributed at random. Percolation theory and the concept of fractal dimensions are related since. Close to percolation threshold, critical phenomena may occur [38].

A pharmaceutical compact is a heterogeneous binary or multiple system constituted by its different components. Monte Carlo simulations can be successfully used to model structures, which can be regarded as the coexistence of clusters of different species (percolating or not, as a function of the volume ratios), because the way in which the physical cluster builds, can be considered random.

In Kosmidis and Argyrakis [22], the authors continued to investigate the benefits of the Monte Carlo method, modelling the drug release from fractal matrices. They consider the possibility of dissolution when the release device, immersed in the gastrointestinal tract fluids, is nonhomogeneously penetrated by these fluids. The gastrointestinal fluids are known to create areas or channels of high-diffusivity inside certain pharmaceutical compacts. For this reason the authors assume that the device can be modelled by an irregular space: i.e. fractal, instead of a homogeneous Euclidean space. This so-called fractal space is represented by a percolation cluster at its critical point, with cyclic boundary conditions. Particles are placed only on the sites of the cluster. They can perform independent random walks on these sites. Alone of the semi-empirical models, the Weibull function is found to be the best fit to the simulation results.

A direct MC study of the transit flow though the gastro-intestinal tract, Kalampokis et al. [21], has been based around the design of a heterogeneous tube model, which simulates the villi of the gastro-intestinal. Authors provided for an empty cylinder with random dendritic-type internal structures, (another example of using a fractal space). The transit flow is simulated using two diffusion models: the blind and the myopic ant, respectively. The two diffusion models involve two types of biased random walk. They place different emphasis on the motion toward the output of the tube. The model seems useful to simulate the transit and absorption process of oral dosage forms in the gastro-intestinal tract.

\section{Multi-particle Monte-Carlo model for a binary soluble system}

We continue to explore the area of Monte-Carlo modelling, in introducing a multi-particle model for another, particular experimental situation. The main target of the model is capture of the phenomenological dynamic behaviour of a relatively complex physical system, consisting of a drug delivery compact, dissolving in a given in vitro environment. Also, the existing models mimic systems which dissolve quite slowly. Here we try to evaluate the possibilities of using Cellular automata $\mathrm{MC}$ to model more rapidly dissolving systems.

We propose a multi-particle Monte Carlo model for binary mixed soluble systems: composed of mixed powders of poorly soluble drug and highly soluble acid excipient. This particular type of compact is obtained by first grinding the two components into powder, mixing the products, and finally compressing them into a final compact, (as described in Healy and Corrigan [18]).

The compact dissolves according to the following main mechanism: at the dissolution of the acid excipient, the $\mathrm{pH}$ of the buffer decreases, affecting the solubility of the other component, eg. ibuprofen (the drug, in this case). The dissolution of ibuprofen is suppressed by the dissolution of the acid excipient (by a pH effect). As the solubility of the excipient is much higher than that of the drug, it is the first to recede from the surface of the compact, permitting the drug to dissolve through the pores left behind.

The in vitro environment is a dissolution test apparatus, consisting of a container filled with the dissolution medium. The compact is situated at the bottom of the apparatus. A paddle is used to stir the buffer solution inside the apparatus, creating a stream, which curves around the obstacle (i.e. the compact), producing a velocity boundary layer around it. Due to the stirred liquid in the apparatus, the mass transport at dissolution happens not only by simple diffusion, but 
by advection ${ }^{8}$ as well: the flow is responsible for carrying away quantities of matter proportional to the velocity.

Our target is to implement a model which captures the essential features necessary to reproduce the complexity observed in experiment:

- Dynamic formation of pores and channels as dissolution takes place.

- Dissolution of the components through the pores and non-uniform suppressing effect of the the acid excipient throughout the pores.

- Given that in the case of the in vitro system the effect of the advection is not negligible, the model specifically has to take into consideration both main mechanisms of mass transport in the real system: diffusion and advection. Hence: accelerated dissolution on the surface, due to advection.

We define the DDS and the release medium around it on a 2D lattice with each site characterised by a local state, as shown in Figure 3. As in Kosmidis et al. [23], we represent the two solid species by virtual particles. The solvent is represented by empty sites. The state of a site is defined by the quantity and type of the particles with which it is filled, i.e. the local concentrations.

The state of any site can evolve in time according to a function which depends on the concentrations of particles at the site itself and those in the local neighbourhood. Zygourakis and Markenscoff [44] also discuss a local concentration approach.

Since the components in the compact have highly different solubilities, they are expected to dissolve at different dissolution rates. In addition, the solubility of a component depends on the local concentrations [17]. In order to model this aspect, we define the maximum allowed concentrations $\left(C_{M A X_{D}}\right.$ and $C_{M A X_{E}}$, measured in terms of numbers of particles per unit of volume, or site) of diffusing particles on the solution sites, proportional to the real solubilities. We allow for more then one particle to hop to an adjacent site, in order to capture the large difference in solubilities between the two components of the disk. If the decision is made for a component to diffuse, then the number of particles chosen to change their position follow a uniform of a normal distribution. For example, in the remainder of this section, the distribution is the following:

$$
X=N(\mu, 1)
$$

where $X$ is the number of particles allowed to modify their position.

\subsection{Quantitative simulations and results}

Qualitative, and then quantitative simulations, have been performed with the above mentioned model. The results agreed quite well with the available experimental results. In the case of the quantitative simulations, we attributed an actual size to to the sites from the lattice. This size equals to the average diameter of the acid excipient powder fragments $(125 \mu \mathrm{m})$. In the experimental data available, the average diameter of the ibuprofen powder fragments is $O\left(10^{2}\right)$ smaller than that of the excipient average diameter of fragments. This is why we assume that the ibuprofen powder simply fills the spaces among the larger excipient bits. If the ratio between the coarsest and the thinner component's average fragment diameters were smaller, it would have been reasonable to take the size of the site equal to the diameter of the smallest component, because there would have been chances that the fragment arrange in a less compact way, letting the possibility of a larger initial porosity.

As stated before, the way to discretise the matter in this model is to fill the sites with "particles". The diffusivities, the densities, the solubilities of the components are used afterwards to calculate the weight of a virtual particle and the loadings of virtual particles per site, in order to set the input parameters to the simulations. For example, to obtain the results presented below we have made the choice that a virtual particle weighs $2 * 10^{-9} \mathrm{~g}$. The Table 1 resumes some of the input

${ }^{8}$ The term of advection refers to the transport of something from one region to another. 


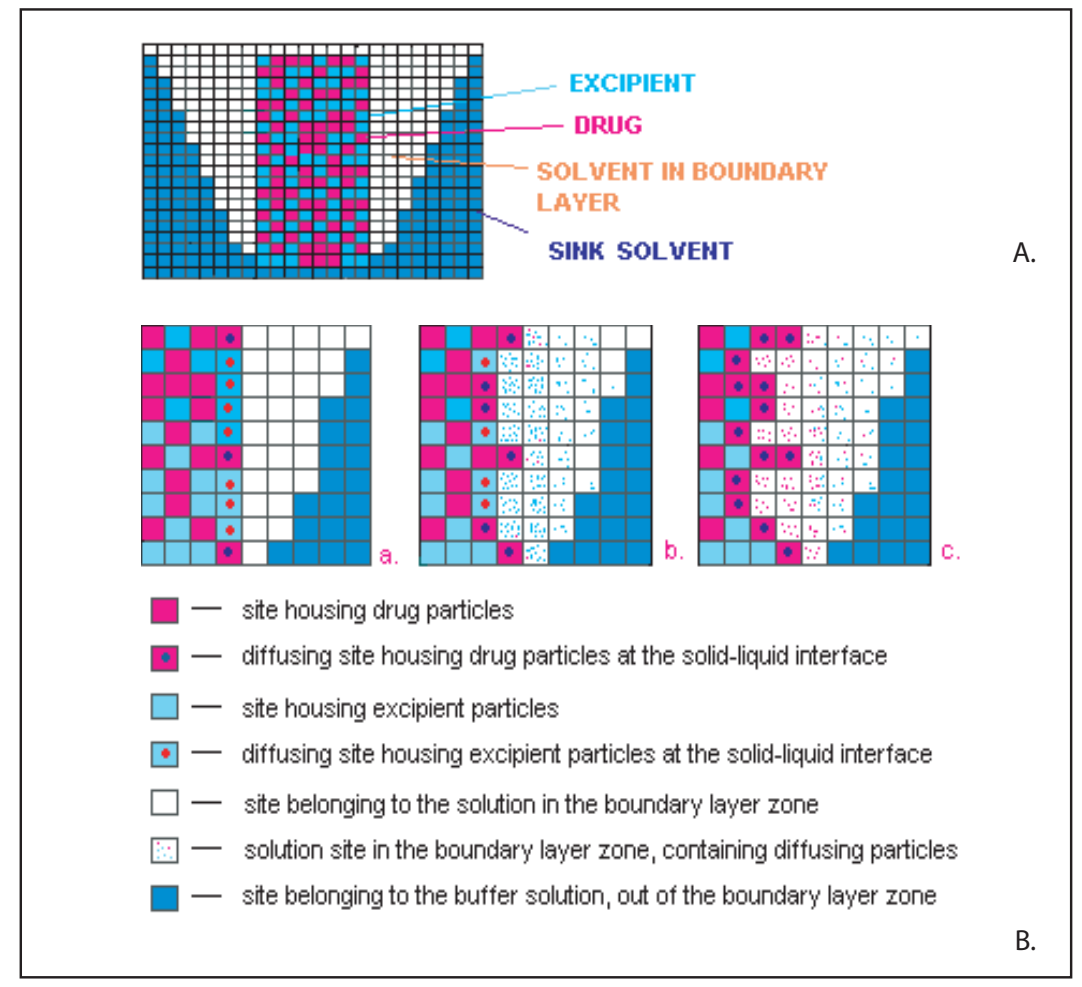

Figure 3: 2D model for a dissolving binary system.

A) Schematic representation of 2D model. The real dimensions are not respected in the picture. B) Simplified model of dissolution of a drug-excipient system. The grid represents a cylindrical tablet in longitudinal cut. The shots show the benefits of this kind of model for simulating cases where pores are formed inside a compact by the quick dissolution of one of the components. The second component has to dissolve through these pores and this process is different from dissolution at the surface. 


\begin{tabular}{|c|c|c|c|c|c|}
\hline & Nb. p. / solid site & Max Nb. p./liquid site & $\mu$ & $\rho\left(\mathrm{mg} / \mathrm{cm}^{3}\right)$ & $D\left(\mathrm{~cm}^{2} / \mathrm{min}\right)$ \\
\hline \hline adipic acid & 1064 & 6 & 1 & 0.98 & $5.155 * 10^{-4}$ \\
\hline citric acid & 933 & 43 & 23 & 1.56 & $4.567 * 10^{-4}$ \\
\hline ibuprofen & 1486 & 841 & 142 & 1.118 & $4.595 * 10^{-4}$ \\
\hline
\end{tabular}

Table 1: Input information for quantitative simulations

a.

Ibuprofen/Succinic acid. (50:50)

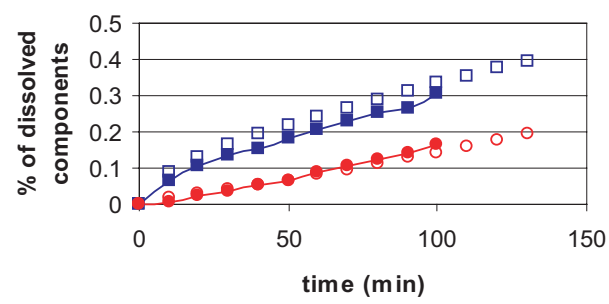

c.

Ibuprofen/Citric acid. (40:60)

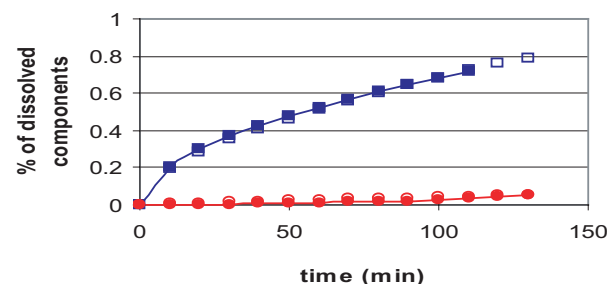

b.

Ibuprofen/Succinic acid. (30:70)

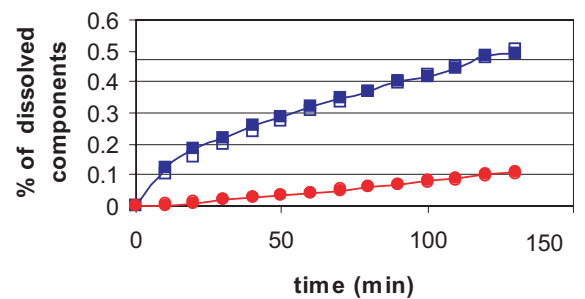

d.

Ibuprofen/Citric acid. (30:70)

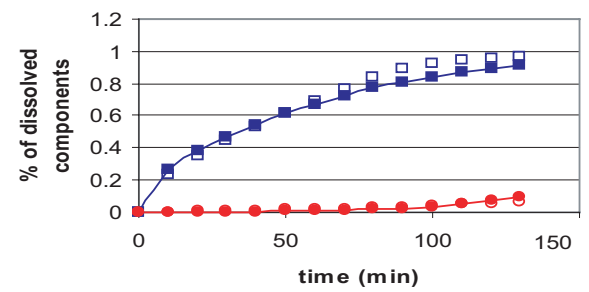

Figure 4: Simulated versus experimental. Continuous lines with filled dots: Dissolution profiles of ibuprofen and excipient from ibuprofen/acid excipient compressed discs. Empty dots: Quantitative simulated results corresponding to respective experimental situations. Red: ibuprofen. Blue: acid excipient. The titles show what are the two components in each experiment and the weight ratio in which they are used.

parameters for the quantitative simulations, as well as some physical characteristics of the real systems.

The transition from Monte Carlo time steps to minutes is done by using Fick's first law:

$$
\frac{d Q}{A d t}=\frac{D C_{s}}{h}
$$

where $d Q$ is the amount of drug released, $h$ is the thickness of the diffusion boundary layer, $D$ is the diffusion coefficient, $C_{s}$ - the solubility and $A$ is the total area through which the diffusion takes place, $[8]$.

The Monte Carlo time step equals to the time needed by a virtual particle of ibuprofen to make it's transition to a adjacent site. We took into consideration the fact that at the surface of the compact the matter passes into the solvent much quicker due to the advection. More particles of ibuprofen, $n_{\text {surface }}$, are allowed to pass into the solution at the surface of the compact in the same interval of time. The experimental profile obtained from the dissolution of an ibuprofen-only tablet was used for the calibration of the distribution of $n_{\text {surface }}$.

Figure 4 compares the simulated data to some of the available experimental dissolution profiles. The first part of the figure ( $a$ and $b$ ) shows the dissolution of the ibuprofen in mixture with the 
Thickness of porous layer as $\mathrm{f}(\mathrm{t})$

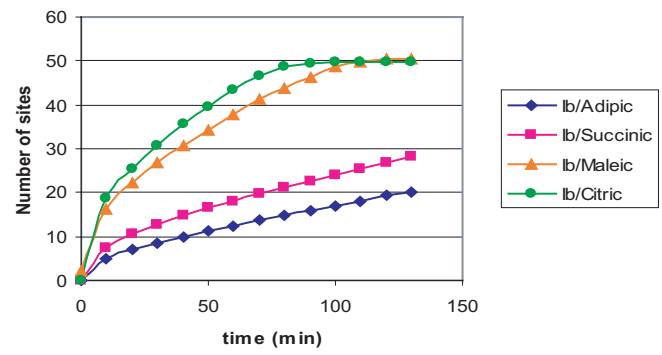

c.

lb/Succinc acid. Thickness of porous layer.

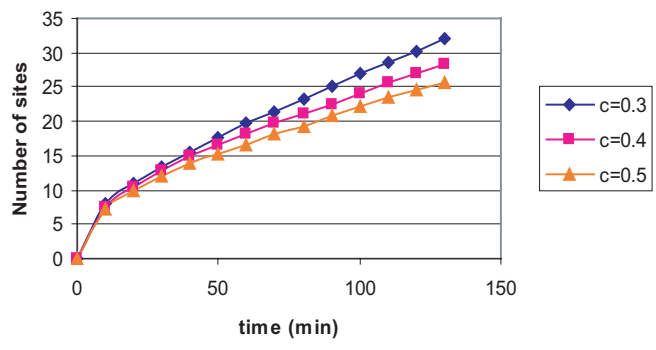

b.

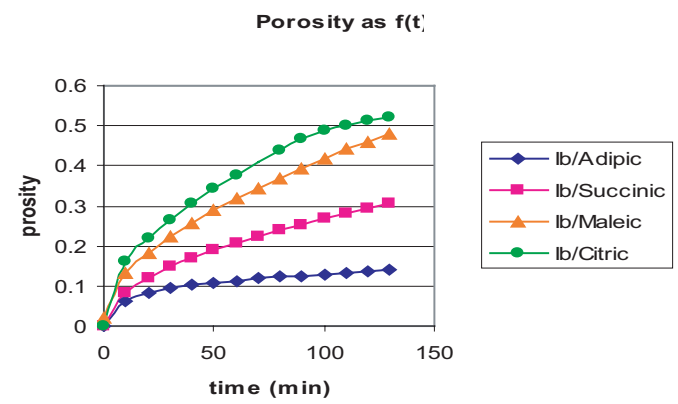

d.

Dissolution profiles of four different excipients

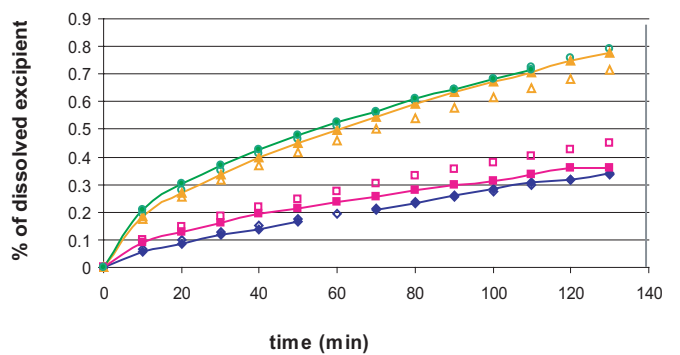

Figure 5: Dynamics of the porous layer. a. Thickness of the porous layer as a function of time. b. Thickness of the porosity as a function of time. c. Thickness of porous layer as a function of time for different initial drug loadings. d. Dissolution profiles of the same four excipients, colour and shape code - the same from the legends of a. and b. Continuous lines with filled dots: experimental. Empty dots: the simulated results. The experimental data appearing on this and previous Figures have been published in $[17,16]$ and have been kindly offered for this comparaison by the authors.

adipic excipient which has a relatively low solubility, $S_{\text {adipic }}=44.4 \mathrm{mg} / \mathrm{ml}$. In Figure 4 , (c and d) the excipient used is the citric acid, $S_{\text {citric }}=883.6 \mathrm{mg} / \mathrm{ml}$.

The good agreement between the simulated and experimental motivates the use of the models to find estimates of other quantities of interest, like the variation of the porosity and thickness of the porous layer, because these influence both drug delivery and mechanical properties of a DDS. The dynamics of these parameters is important to be known in many other applications. For example, in the cases of therapeutical implants, the designers endeavour to find a good compromise between the drug delivery properties of the implant and it's mechanical properties, as the components dissolve from the compact and form channels and pores. Figure $5(\mathrm{a}, \mathrm{b})$ shows the dynamics of the porous layer in the case of mixtures of ibuprofen and four different acid excipients. The thickness of porous layer is given in sites, but can be easily converted in $\mathrm{cm}$ because the diameter of the site is known. The porosity is defined as the ratio between the number of sites labelled as pores and the total number of sites currently belonging to the device. It can be observed that both porosity and thickness of the porous layer have the same shape as the receding acid excipient dissolution profiles (compare with Figure 5, d.).

We report a better description of the above presented problem, implementation details and more results of this model in Part2, the companion paper on our model. 


\section{Conclusions}

The results obtained by different Monte Carlo modelling approaches reported here, in particular where these draw on the computing power of high performance computing systems, have shown considerable promises for the area of drug design and drug formulation. Further work is clearly needed, especially to counter difficulties in calibration of the MC models with real systems, in order to obtain quantitative results.

The advantage of the MC models is that they can address diversity in general and offer a straightforward means of specifying and describing micro-structural situations and relationships, thus permitting detailed study of the phenomena occurring at dissolution, including the different types of interactions between the components of a dissolving compact, dissolution through a system of channels and pores, establishment of dissolution boundary layers and so on. In addition, MC models can be easily modified and adapted to mimic a wide range of physical situations corresponding to various drug delivery systems.

\section{References}

[1] http://wikipedia.org, 2005.

[2] I. Antal, R. Zelkö, N. Röczey, J. Plachy, and I. Rácz. Dissolution and diffuse reflectance characteristics of coated theophylline particles. International Journal of Pharmaceutics, 155:83-89, 1997.

[3] L. P. Balant and M. Gex-Fabry. Modelling during drug development. European Journal of Pharmaceutics and Biopharmaceutics, 50:13-26, 2000.

[4] I. Caraballo. Ultrasound-assisted compression. A new perspective for the preparation of controlled release systems. Leader for Chemist, 2004. http://www.cesil.com/leaderforchemist/chemist.htm.

[5] X. Chen, W. Chen, A. H. Hical, B.-C. Shen, and L. T. Fan. Stochastic modeling of controlleddrug release. Biochemical Engineering Journal, 2:161-177, 1998.

[6] Y. Chen, T. McCall, A. Baichwal, and M. Meyer. The application of an artificial neural network and pharmacokinetic simulations in the design of controlled-release dosage forms. $J$. Control. Release, 59:33-41, 1999.

[7] B. Chopard and M. Droz. Cellular Automata Modeling of Physical Systems. Cambridge University Press, 1998.

[8] P. Costa and J. M. S. Lobo. Modeling and comparison of dissolution profiles. European Journal of Pharmaceutical Sciences, 13:123-133, 2001.

[9] J. Cranck. The Mathematics of Diffusion. Clarendon Press, Oxford, 1975.

[10] M. Crane, L. Crane, A. M. Healy, O. I. Corrigan, K. M. Gallagher, and L. McCarthy. A Pohlhausen solution for the Mass Flux From a Multi-layered Compact in the USP Drug Dissolution Apparatus. Simulation Modelling Practice and Theory (SIMPAT), 2004.

[11] A. Göpferich. Modeling of polymer erosion in three dimensions: rotationally symmetric devices. AIChE, 41(10), 1995.

[12] A. Göpferich. Bioerodible implants with programmable drug release. Journal of Controlled Release, 44:271-281, 1997.

[13] A. Göpferich. Erosion of composite polymer matrices. Biomaterials, 18:397-403, 1997. 
[14] A. Göpferich and R. Langer. Modeling monomer release from bioerodible polymers. Journal of Controlled Release, 33:55-69, 1995.

[15] M. Grassi, I. Colombo, and R. Lapasin. Drug release from an ensemble of swellable crosslinked polymer particles. Journal of Controlled Release, 68:97-113, 2000.

[16] A. M. Healy. Investigations of the dissolution mechanisms of acidic drug-excipient compacts. PhD thesis, University of Dublin, Trinity College, 1995.

[17] A. M. Healy and O. I. Corrigan. Predicting the dissolution rate of ibuprofen-acidic excipient compressed mixtures in reactive media. International Journal of Pharmaceutics, 84:167-173, 1992.

[18] A. M. Healy and O. I. Corrigan. The influence of excipient particle size, solubility and acid strength on the dissolution of an acidic drug from two-component compacts. International Journal of Pharmaceutics, 143:211-221, 1996.

[19] D. Higdon, H. Lee, and Z. Bi. A Bayesian approach to characterizing Uncertainty in inverse problems using coarse and fine scale information, 2001.

[20] J. P. Huelsenbeck, F. Ronquist, and B. Hall. Mrbayes: A program for the Bayesian inference of phylogeny, 2001.

[21] A. Kalampokis, P. Argyrakis, and P. Macheras. Heterogeneous tube model for the study of small intestinal transit flow. Pharmaceutical Research, 16(1), 1999.

[22] K. Kosmidis and P. Argyrakis. Fractal kinetics in drug release from finite fractal matrices. Journal of Chemical Physics, 119(12), 2000.

[23] K. Kosmidis, P. Argyrakis, and P. Macheras. A reappraisal of drug release laws using Monte Carlo simulations: the prevalence of the Weibull function. Pharmaceutical Research, 20, 2003.

[24] K. Kosmidis, E. Rinaki, P. Argyrakis, and P. Macheras. Analysis of case ii drug transport with radial and axial release from cylinders. International Journal of Pharmaceutics, 254, 2003.

[25] D. P. Landau and K. Binder. Monte Carlo Simulations in Statistical Physics. Cambridge University Press, 2000.

[26] S.-J. E. Lee and A. K. Chakraborty. Sequence dependence of polymer dynamics in quenched disordered media: Weak attraction facilitates transport. Journal of Chemical Physics, $117(23), 2002$.

[27] H. Leuenberger, B. Rohera, and C. Haas. Percolation theory - a novel approach to solid dosage form design. IJP, 38:109-115, 1987.

[28] K. Mosegaard and M. Sambridge. Monte Carlo analysis of inverse problems. Inverse Problems, 18:R29-R54, 2002.

[29] B. Narasimhan. Mathematical models describing polymer dissolution: consequences for drug delivery. Advanced Drug Delivery Reviews, 48:195-210, 2001.

[30] Z. Ramtoola and O. I. Corrigan. Dissolution characteristics of benzoic acid and salicylic acid mixtures in reactive media. Drug Development and Industrial Pharmacy, 13:9-11, 1987.

[31] S. B. Santra, B. Sapoval, P. Barboux, and F. Devreux. Pseudo-equilibrum between a random system and a solution: a Monte-Carlo study of glass dissolution in water. C. R. Acad. Sci. Paris, 326:129-134, 1998. 
[32] J. Siepmann. Mathematical modeling of controlled drug delivery. Advanced Drug Delivery Reviews, 48:137-138, 2001. Preface.

[33] J. Siepmann and A. Göpferich. Mathematical modeling of bioerodible, polymeric drug delivery systems. Advanced Drug Delivery Reviews, 48:229-247, 2001.

[34] J. Siepmann, H. Kranz, N. Peppas, and R. Bodmeier. Calculation of the required size and shape of hydroxypropyl methylcellulose matrices to achieve desired drug release profiles. International Journal of Pharmaceutics, 201:151-164, 2000.

[35] J. Siepmann and N. A. Peppas. Modeling of drug release from delivery systems based on hydroxypropyl methylcellulose (HPMC). Advanced Drug Delivery Reviews, 48:139-157, 2001.

[36] N. Sirisuth and N. D. Eddington. In-vitro-in-vivo correlation definitions and regulatory guidance, 2003.

[37] R. Srinivasan. Application of the Monte Carlo method to the dissolution of a polycrystalline solid. Materials Letters, 31:5-9, 1997.

[38] D. Stauffer and A. Aharony. Cluster numbers, in Introduction to Percolation Theory, 2nd Edn. Taylor |\& Francis, London and Philadelphia, 1992.

[39] Y. Sun, Y. Peng, Y. Chen, and A. J. Shukla. Application of artificial neural networks in the design of controlled release drug delivery systems. Advanced Drug Delivery Reviews, 55:1201-1215, 2003. Preface.

[40] Takahara, K. Takayama, and T. Nagai. Multi-objective simultaneous optimization technique based on an artificial neural network in sustained release formulations. J. Control. Release, 49:11-20, 1997.

[41] K. Takayama, J. Takahara, M. Fujikawa, H. Ichikawa, and T. Nagai. Formula optimization based on artificial neural networks in transdermal drug delivery. J. Control. Release, 62:161170, 1999.

[42] Vaithiyalingam and M. Khan. Optimization and characterization of controlled release multiparticulate beads formulated with a customized cellulose acetate butyrate dispersion. IJP, 234:179-193, 2002.

[43] R. E. Williford, D. R. Baer, J. E. Amonette, and A. S. Lea. Dissolution and growth of (1 014 ) calcite in flowing water: estimation of back reaction rates via kinetic Monte Carlo Simulations. Journal of Crystal Growth, 262:503-518, 2004.

[44] K. Zygourakis and P. A. Markenscoff. Computer-aided design of bioerodible devices with optimal release characteristics: a cellular automata approach. Biomaterials, 17:125-135, 1996. 